\title{
Syphilis serology in patients with primary syphilis and non-treponemal sexually transmitted diseases in southern Africa
}

\author{
A Sischy, F da L'Exposto, Y Dangor, H G Fehler, F Radebe, D D Walkden, S D Miller, \\ R C Ballard
}

\begin{abstract}
The reactivity of a non-specific reagin (RPR) test and a specific treponemal (FTA-ABS) test were determined in 21 patients with primary syphilis, 430 patients with proven non-treponemal genital ulcerations and 719 patients with acute urethritis presenting at a clinic for sexually transmitted diseases in southern Africa. Excluding those 21 cases of primary syphilis, 358 of 1149 tests performed (31\%) were found to be reactive by at least one test. The rate of false positive RPR tests was very low (0.02\%). Significantly higher rates of seropositivity were detected in patients with genital ulcerations than in patients with acute urethritis. The highest rates were detected among patients with proven lymphogranuloma venereum (34\% RPR positive, FTA-ABS positive; $19 \%$ RPR negative, FTA-ABS positive). The geometric mean titres (GMT) of positive RPR tests in non-treponemal infections were found to be lower than in darkfield positive cases of genital ulcer disease.
\end{abstract}

\section{Introduction}

Syphilis remains a major public health problem in many developing countries ${ }^{12}$ and has recently reemerged as a significant heterosexual disease in the United States of America ${ }^{34}$ and Europe. ${ }^{56}$ In many societies the coexistence of two or more sexually transmitted infections poses both diagnostic and therapeutic problems to clinicians working in the field. Whilst conducting studies on sexually transmitted diseases (STDs) in southern Africa, it was realised that a large number of patients presenting with non-treponemal STDs also had reactive

Emergent Pathogen Research Unit of the South African Medical Research Council, Department of Microbiology, School of Pathology of the University of the Witwatersrand and South African Institute for Medical Research, Johannesburg, South Africa. A Sischy, F da L'Exposto, Y Dangor, H G Fehler, F Radebe, D D Walkden, S D Miller, R C Ballard serological tests for syphilis. In order to establish the significance of these findings, a study was initiated to determine if positive syphilis serology was associated with the presence of any particular non-treponemal STD and also to determine whether these serological reactions were of clinical importance.

\section{Materials and methods}

A total of 1170 black mineworkers presenting with acute urethritis or genital ulceration at a clinic for sexually transmitted diseases at the Leslie Williams Memorial Hospital, Carletonville, Transvaal, South Africa, were included in this study. In each case a clinical examination was performed and appropriate specimens were taken for microbiological investigations.

In all cases of acute urethritis a cotton-tipped meatal swab was used to collect urethral exudate. This was rolled onto two microscope slides and stained by Gram's method and by using a modified Giemsa stain (Dif-Quik, Harleco) for cytology. Patients were included in the study as cases of acute urethritis if there were $\geqslant 4$ polymorphonuclear leucocytes seen per $\times 1000$ high power microscope field. A diagnosis of gonococcal urethritis was based on the finding of Gram-negative intracellular diplococci and the subsequent isolation of Neisseria gonorrhoeae on modified New York City medium. Cases of non-gonococcal urethritis were diagnosed by exclusion of gonorrhoea.

In cases of genital ulcer disease (GUD) the largest ulcer was selected for investigation. Material from the ulcer base was examined by darkfield microscopy for Treponema pallidum. Giemsa-stained smears were examined for the presence of Donovan bodies indicative of infection with Calymmatobacterium granulomatis. Calcium alginate swabs were used to collect material from the bases of the lesions for the isolation of Haemophilus ducreyi, Chlamydia trachomatis and herpes simplex virus (HSV). Isolation of $H$. ducreyi was performed by inoculating samples directly onto two selective media as described previously. ${ }^{78} C$. trachomatis and HSV were isolated by using cycloheximide-treated McCoy cells ${ }^{9}$ and human embryo lung fibroblasts respectively. In each 
case a serum specimen was obtained for the detection of type-specific antichlamydial antibody using the modified microimmunofluorescence technique. ${ }^{10}$ Broadly cross-reactive antibodies at titres $\geqslant 1: 256$ were considered diagnostic for lymphogranuloma venereum (LGV) where the clinical evidence also supported the diagnosis. A diagnosis of syphilis was established on the basis of a positive darkfield examination and absence of any laboratory evidence of infection by other aetiological agents of GUD. Serological evidence of syphilis was sought in all cases of acute urethritis and genital ulcer disease using both the non-specific rapid plasma reagin (RPR) (Becton Dickinson Microbiology Systems) and specific fluorescent treponemal antibody absorbed (FTA-ABS) (Wellcome Diagnostics) tests using standard techniques. Sera were considered reactive in the RPR test if flocculation occurred in the presence of neat or subsequent dilutions of serum.

Subsequently, the results of syphilis serology were grouped into four categories, namely, those sera which were both RPR and FTA-ABS positive, those which were RPR negative and FTA-ABS positive, those which were RPR positive and FTA-ABS negative and those which were both RPR and FTAABS negative.

\section{Results}

During the course of the study, 21 cases of syphilis were diagnosed on the basis of darkfield microscopy and by exclusion of other aetiological agents of GUD. Of these, $15(71 \%)$ exhibited reactivity by both the RPR and FTA-ABS tests. Four (19\%) were found to be reactive by the FTA-ABS test alone and two $(10 \%)$ were negative by both tests. Although the latter patients could reflect cases of false positive darkfield microscopy, the patients were not available for follow-up examination for repeat serological testing to detect seroconversion. A further two patients found to be darkfield-positive had micro- biological evidence of concomitant infection with $H$. ducreyi and a further patient found to harbour HSV, was also darkfield-positive. While only one patient with chancroid was found to be serologically positive by both the RPR (titre =4) and FTA-ABS tests the remaining two cases were reactive by the FTA-ABS test alone.

The patterns of reactivity of both non-specific and specific serological tests for syphilis in patients presenting with proven non-treponemal STDs are shown in table 1 . Overall, 358 of 1149 tests performed on these patients $(31 \%)$ were found to be reactive by at least one test, while only two false positive RPR tests (RPR titres neat and 2, FTA-ABS negative) were detected during the study $(0.02 \%)$. Significantly higher rates of seroreactivity were detected in patients with GUD [98 of $430(23 \%)$ ] than in patients with acute urethritis [78 of 719 $(11 \%)],\left[\chi^{2}=28.67, p<0,0001\right)$ but no significant difference in reactivity was detected between those patients presenting with gonorrhoea and those with NGU. The highest seropositivity rate was observed amongst those patients diagnosed as having LGV (34\%).

The geometric mean titres (GMT) of reactive RPR tests detected in cases of proven syphilis and other non-treponemal STD's are shown in table 2. No significant differences in GMT were demonstrated between all non-treponemal STDs (range 5-9). However, a higher GMT was demonstrated in cases of proven syphilis when compared with the nontreponemal STDs and a significantly higher proportion of cases proved to be reactive $(p<0.00001$ Fisher's exact test).

If an RPR titre of $\geqslant 8$, confirmed by a FTA-ABS test was considered as the sole criterion for the diagnosis of syphilis, then only 12 of 21 cases of darkfield positive genital ulcers $(57 \%)$ would have been diagnosed and nine cases would have remained undiagnosed. Using this same criterion, only 46 of

Table 1 Reactivity of non-specific (RPR) and specific (FTA-ABS) tests for syphilis in patients with non-treponemal STDs

\begin{tabular}{|c|c|c|c|c|c|c|}
\hline Test & Gonorrhoea (\%) & $N G U(\%)$ & $\begin{array}{l}\text { Genital } \\
\text { herpes (\%) }\end{array}$ & Chancroid (\%) & $L G V(\%)$ & Total (\%) \\
\hline $\begin{array}{l}\text { RPR + ve } \\
\text { FTA-ABS + ve }\end{array}$ & $64(11)$ & $14(11)$ & $6(24)$ & $57(19)$ & $35(34)$ & $176(15 \cdot 31)$ \\
\hline $\begin{array}{l}\text { RPR -ve } \\
\text { FTA-ABS + ve }\end{array}$ & $77(13)$ & $27(20)$ & $2(8)$ & $54(18)$ & $20(19)$ & $180(15 \cdot 67)$ \\
\hline $\begin{array}{l}\text { RPR + ve } \\
\text { FTA-ABS - ve }\end{array}$ & $2(0.3)$ & 0 & $\mathbf{0}$ & 0 & 0 & $2(0.02)$ \\
\hline $\begin{array}{l}\text { RPR -ve } \\
\text { FTA-ABS - ve }\end{array}$ & $443(76)$ & $92(69)$ & $17(68)$ & $191(63)$ & $48(47)$ & $791(68.84)$ \\
\hline Total & 586 & 133 & 25 & 302 & 103 & 1149 \\
\hline
\end{tabular}

NGU = non-gonococcal urethritis.

LGV = lymphogranuloma venereum. 
Table 2 Percentage reactivity and geometric mean titres (GMT) of RPR tests in patients with syphilis and other non-treponemal STDs

\begin{tabular}{llll}
\hline STD & $\begin{array}{l}\text { No sera reactive/ } \\
\text { Total no tested }\end{array}$ & $\%$ & GMT \\
\hline Syphilis & $15 / 21$ & 71 & 25 \\
Gonorrhoea & $64 / 586$ & 11 & 9 \\
NGU & $14 / 133$ & 11 & 6 \\
Herpes & $6 / 25$ & 24 & 5 \\
Chancroid & $57 / 302$ & 19 & 8 \\
LGV & $35 / 103$ & 34 & 5 \\
\hline
\end{tabular}

430 cases of non-treponemal genital ùlcers $(10 \cdot 7 \%)$ and 42 of 719 cases of acute urethritis $(5.8 \%)$ would have been diagnosed as syphilis.

\section{Discussion}

This study clearly indicates that the establishment of a diagnosis of syphilis in individual cases of GUD may be problematic especially in areas where the prevalence of venereal syphilis is high. While darkfield microscopy will continue to be the rapid diagnostic method of choice, problems encountered with the technique ${ }^{1112}$ necessitate reliance on serological methods to diagnose the disease in most developing countries. Thus, in the small series of darkfieldpositive cases described in this study, 15 of $21(71 \%)$ exhibited reactivity by both the RPR and FTA-ABS tests and a further four (19\%) were found to be reactive by the FTA-ABS test alone. These results, together with the finding of two cases which were non-reactive by both tests, indicate that approximately one quarter of cases would not have been detected if a diagnosis had been based on routine screening with the RPR test alone. While additional use of the FTA-ABS test would have detected a further $19 \%$ of primary syphilis cases, the finding of a reactive FTA-ABS test together with a negative RPR test in patients with other STDs poses an interpretive dilemma since these patients could have very early syphilis or previously treated early disease. Likewise, a similar scenario could be painted for patients who were found to be reactive by both tests since many of them had RPR titres $\leqslant 8$. A higher proportion of darkfield-positive cases of GUD than any other diagnostic category were found to have titres $\geqslant 8$, indicating that the patterns of reactivity detected amongst cases of non-treponemal disease could possibly reflect adequately or inadequately-treated previous infection or recently acquired infection rather than untreated active concomitant treponemal disease.

As a result of the low rate $(0.02 \%)$ of false positive RPR tests detected in this study, there appears to be little value in routinely confirming the specificity of reactive RPR tests with the FTA-ABS test in this population. Since the FTA-ABS test may become positive three weeks before the RPR test, the value of the FTA-ABS test may be in the detection of a minority of cases of early syphilis. In contrast, another specific test, the Treponema pallidum haemagglutination assay (TPHA) becomes positive around the same time as the RPR test. ${ }^{13}$ Performance of the TPHA test in addition to the RPR test would yield little additional information in this patient group.

Venereal syphilis remains a significant problem in southern Africa accounting for 15-35\% of GUD. ${ }^{714}$ In this study, positive tests for syphilis were detected more frequently in patients presenting with nontreponemal genital ulcerations than in patients with acute urethritis. It is possible that previous infection with syphilis may have disrupted epithelial integrity, allowing subsequent invasion by other causative agents of GUD. Since a diagnosis of GUD is frequently made following prostitute contact, concomitant acquisition of syphilis and other causes of GUD may also be a factor. The highest rate of seropositivity was found in patients with an established diagnosis of LGV. The reasons for this finding remain unclear. However, the longer incubation period of LGV when compared to other causes of GUD and delay in presentation may allow development of reactive serological tests for syphilis in more cases of LGV than other causes of GUD. It is also possible that there is a geographical association between syphilis and LGV, because LGV has largely been documented in a distinct geographical region of southern Africa ${ }^{15}$ which may also have a disproportionately high rate of syphilis. In contrast, the rates of seropositivity detected in cases of acute urethritis could reflect the baseline rate in this population of sexually active patients exposed to STDs in general.

These findings clearly have important implications for the provision of appropriate therapy to patients presenting with STDs in southern Africa. Since darkfield microscopy is only performed in a minority of centres owing to lack of technical expertise, the syphilitic status of patients presenting with GUD and positive syphilis serology is not known with certainty. It is therefore suggested that they should be treated routinely for treponemal disease in addition to the therapy normally provided for their particular infection. However, since the reactivity of their serological tests does not give an indication of whether they have early or late disease, it is advisible to provide adequate therapy to cover for late disease.

Address for correspondence: Dr A Sischy, Department of Microbiology, SAIMR, PO Box 1038, Johannesburg 2000, South Africa.

1 Osoba AO. Sexually transmitted diseases in tropical Africa. $\mathrm{Br} J$ Venereal Dis 1981;57:89-94.

2 Dupont A, Schrijvers D, Delaporte E. et al. Seroprevalence de la syphilis dans des populations urbaines et semi-rurales du Gabon. Bull Soc Pathol Exot Filiales 1988;81:699-704. 
3 Rolfs RT, Cates W. The perpetual lessons of syphilis. Arch Dermatol 1989;125:107-9.

4 Centers for Disease Control. Syphilis and congenital syphilisUnited States, 1985-1988. MMWR 1988;37(32):486-9.

5 van den Hoek JAR, van der Linden MMD, Coutinho RA. Increase of infectious syphilis among heterosexuals in Amsterdam: its relationship to drug use and prostitution. Genitourin Med 1990;66:31-32.

6 Tang A, Barlow D. Resurgence of heterosexually acquired early syphilis in London. Lancet 1989;ii:166-7.

7 Duncan MO, Bilgeri YR, Fehler HG, Ballard RC. The diagnosis of sexually acquired genital ulcerations in black patients in Johannesburg. S Afr J Sex Transm Dis 1981;1:20-23.

8 Nsanze H, Fast MV, D'Costa L et al. Genital ulcers in Kenya: a clinical and laboratory study. $\mathrm{Br} J$ Venereal Dis 1981;57: 378-81.

9 Ripa KT, Mådh P-A. Cultivation of Chlamydia trachomatis in cycloheximide treated McCoy cells. J Clin Microbio 1977;6:328-31.

10 Treharne JD, Darougar S, Jones BR. Modification of the microimmunofluorescence test to provide a routine serodiag- nostic test for chlamydial infection. $J$ Clin Pathol 1977;30: 510-7.

11 Anderson J, Mindel A, Tovey SJ, Williams P. Primary and secondary syphilis, 20 years experience 3 : diagnosis, treatment, and follow-up. Genitourin Med 1989;65:239-43.

12 Adler MW. ABC of sexually transmitted diseases. Syphilis diagnosis and management. BMJ 1984;288:551-3.

13 Hart G. Syphilis tests in diagnostic and therapeutic decision making. Ann Intern Med 1986;104:368-76.

14 Coovadia YM, Kharsany A, Hoosen A. The microbial aetiology of genital ulcers in black men in Durban, South Africa. Genitourin Med 1985;61:266-9.

15 Piot P, Ballard RC, Fehler HG, van Dyck E, Ursi JP, Meheus AZ. Isolation of Chlamydia trachomatis from genital ulcerations in southern Africa. In: Märdh P-A, Homes KK, Oriel JD, Piot P, Schachter J, eds. Chlamydial Infections. Amsterdam: Elsevier Biomedical Press, 1982;115-8.

Accepted for publication 20 November 1990 